\title{
MODELO DINÁMICO Y CONTROL NO LINEAL PARA UN CONVERTIDOR DE ENERGÍA DE LAS CORRIENTES MARINAS DE PRIMERA GENERACIÓN Y DOS GRADOS DE LIBERTAD
}

\author{
E. Segura, R. Morales \\ Escuela de Ingenieros Industriales de Albacete, Universidad de \\ Castilla-La Mancha, 02071 Albacete, Spain. eva.segura, rafael.morales@uclm.es \\ José A. Somolinos \\ -GIT-ERM- ETS I Navales. Universidad Politécnica de Madrid, 08040 Madrid. \\ joseandres.somolinos@upm.es
}

\begin{abstract}
Resumen
Diversos fabricantes han desarrollado dispositivos con los que explotar energía de las corrientes marinas en zonas donde la profundidad no supera los 40 metros. Estos dispositivos son denominados TEC de $1^{a}$ generación. Las tareas de mantenimiento requieren extraerlos de su base a la superficie del mar y retornar a su base. Es posible automatizar las maniobras de emersión/inmersión y se han presentado previamente diversos trabajos. Este trabajo presenta el modelo dinámico de control más simple posible para un TEC accionado por fuerzas hidrostáticas. Se propone un sistema de control PD con compensación de los términos no lineales que garantiza estabilidad asintótica y global, además de un buen comportamiento en movimiento.
\end{abstract}

Palabras clave: TEC (Tidal Energy Converter), Energías Renovables Marinas, Modelado dinámico, Control no lineal.

\section{INTRODUCCIÓN}

La energía de las corrientes es un recurso renovable de gran interés en aras a proporcionar un gran valor energético en el mercado eléctrico futuro gracias sobre todo, a su predictibilidad [3], [5]. Algunas de las principales oportunidades y beneficios de esta fuente de energía incluyen independencia energética, creación de puestos de trabajo, decarbonización, etc, dentro del mix energético global. Diversos fabricantes están desarrollando dispositivos con los que explotar energía hidrocinética de las corrientes marinas en zonas donde las profundidades no superan $\operatorname{los} 40 \mathrm{~m}$ [1],[2],[13]. La Figura 1 muestra un ejemplo de TEC basado en rotor abierto. Estos dispositivos están normalmente situados sobre una base que es fija al fondo marino con diversos sistemas de fondeo, y son denominados de $1^{\text {a }}$ generación.

Es necesario tomar en consideración y promover las tecnologías oceánicas para aumentar su potencial de explotación energética. Ello depende de su viabilidad económica y técnica, supervivencia de los dispositivos ante tormentas y otros fenómenos del mar, y accesibilidad para mantenimiento. Estos retos pueden alcanzarse reduciendo costes de instalación y sobre todo de mantenimiento, automatizando maniobras de emersión e inmersión, lo que puede acelerar la implantación de estos sistemas [11],[12]. Una de las posibles opciones implica el uso de sistemas de gestión de lastre para generar fuerzas hidrostáticas verticales, permitiendo que los movimientos de emersión e inmersión de los dispositivos puedan ser automatizados.

Algunos sistemas de control para estas maniobras han sido propuestos previamente por el grupo GITERM, en base al mencionado control de agua de lastre de sus accionamientos. [4] obteniéndose modelos dinámicos de cuerpos sumergidos con diversas formas. [4],[8]. Este trabajo resume un modelo dinámico muy simple y presenta un control no lineal para un TEC de $1^{\mathrm{a}}$ generación con dos grados de libertad.

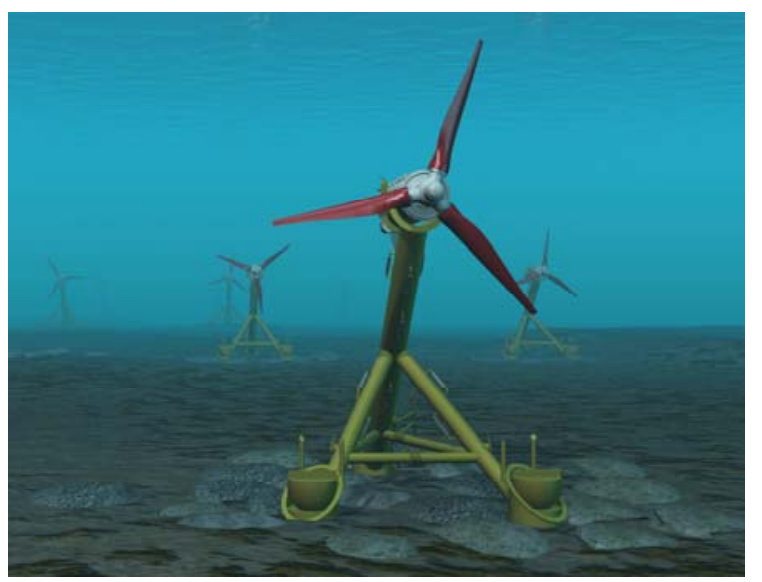

Figura 1. Parque de TEC de primera generación

(Andriz [2])

El trabajo está organizado como sigue: en la sección 2 se resume el mencionado modelo dinámico para dos grados de libertad, la obtención del sistema de 
control se presenta en el apartado 3, mientras diversos resultados de simulación son expuestos en el apartado 4 y algunas conclusiones y trabajos futuros son resaltados en el apartado 5 .

(a)

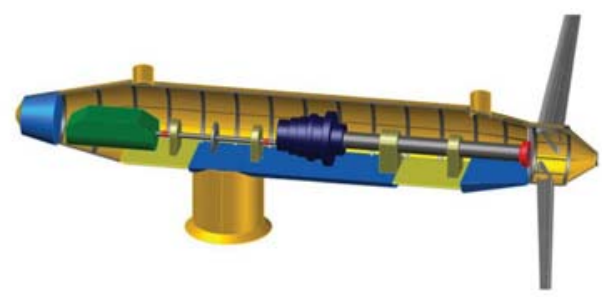

(b)

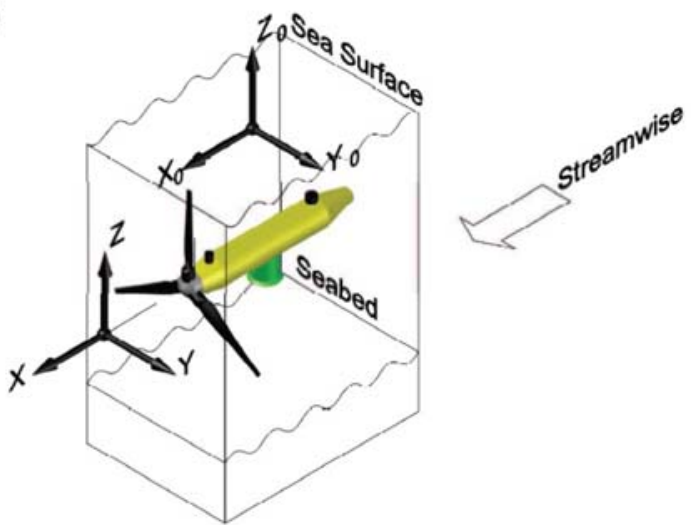

Figura 2. (a) Distribución de equipos en un TEC modificado. (b) Sistemas de referencia

\section{MODELO DINÁMICO DE UN TEC DE 1ª GENERACIÓN}

A partir de la disposición de equipamiento del TEC mostrado en la Figura 2(a) se resaltan las modificaciones y los elementos que han sido añadidos para llevar a cabo las maniobras: a) pueden apreciarse los tanques de lastre, b) se ha modificado la longitud del TEC para obtener flotabilidad neutra con los tanques al 50\%; c) no se detalla la cámara de máquinas para el sistema de vaciado y llenado de tanques. El modelo que aquí se resume es suficientemente preciso para describir el comportamiento del dispositivo $\mathrm{y}$, a la vez, suficientemente simple para poder incluirse en la ley de control. Los sistemas de referencia vienen dados por la norma (IEC-TC214) [7]. Un sistema fijo $\left\{\mathbf{S}_{0}\right\}$ situado en el nivel del mar y en la vertical del TEC y un sistema móvil $\{\mathbf{S}\}$ con sus plano xy paralelos al nivel del mar. Con respecto a este último se mide la orientación del TEC representada por un sistema local $\left\{\mathbf{S}_{\mathrm{L}}\right\}$ en el centro de carena del TEC. Un modelo dinámico práctico se obtiene considerando la parte móvil del dispositivo como un cilindro. Está provisto de dos grados de libertad: la profundidad $\mathrm{z}(\mathrm{t})$ y la rotación alrededor del eje y del sistema $\{\mathrm{S}\}$, $\theta(\mathrm{t})$.

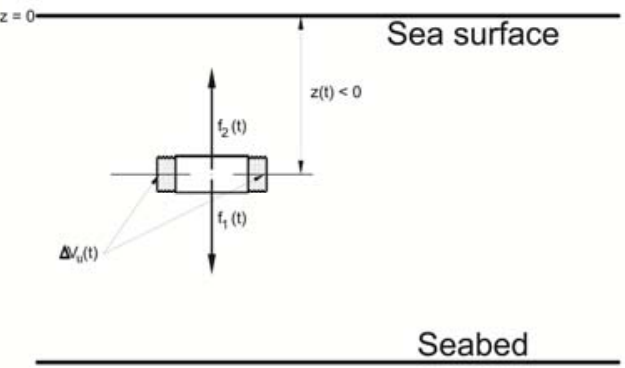

(b)

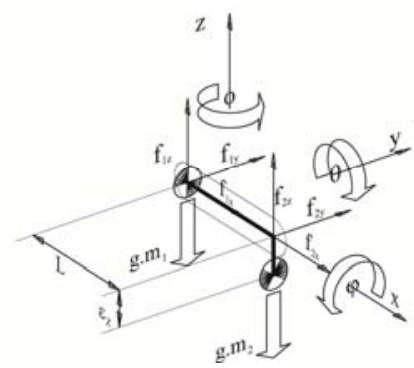

Figura 3. (a) Principales variables. (b) Modelo de dos masas concentradas

La Figura 3(a) ofrece una representación esquemática de las principales magnitudes del cuerpo cilíndrico cuando los accionamientos utilizados para producir fuerzas verticales son concebidos como accionamientos de incremento de volumen. Se suponen dos masas concentradas en sus extremos (m $=\mathrm{m}_{1}+\mathrm{m}_{2}$ ), si bien la distribución interna de masas debe garantizar la estabilidad durante la etapa de flotación en horizontal, y los puntos de aplicación de las fuerzas han sido desplazados ligeramente de los puntos de simetría. La Figura 3(b) muestra la situación de las dos masas, las fuerzas hidrostáticas aplicadas y el criterio de signos utilizado en las magnitudes. Este desplazamiento es relativamente pequeño $\left(\varepsilon_{\mathrm{z}}>0\right.$ en comparación con el diámetro del cilindro) y se modela como un desplazamiento vertical en la masa 2. En primer lugar, la dinámica traslacional se obtiene como sigue:

$$
\left(m_{1}+m_{2}+m_{\text {Add }}\right) \ddot{z}+\nu \cdot \operatorname{sign}(\dot{z}) \cdot \dot{z}^{2}=f_{g}+f_{b}
$$

Donde el subíndice $i$ representa el número de masa en la que se sitúa cada accionamiento, $\mathrm{m}_{\mathrm{Add}}=$ $\mathrm{m}_{\text {Add }}\{\mathrm{z}(\mathrm{t}), \theta(\mathrm{t})\}$ representa la masa añadida. Se propone que este parámetro dependa sólo de la profundidad y orientación del dispositivo, ignorando su dependencia con la velocidad relativa del fluido, tipo de superficie, etc. El coeficiente $v$ denota fricción, mientras que $\mathrm{fg}$ y $\mathrm{fb}$ son las únicas fuerzas aplicadas al dispositivo en ausencia de otras como las fuerzas medioambientales, fondeo etc. que son consideradas como perturbaciones externas. Todas las fuerzas y variables son computadas como sigue: 


$$
\begin{aligned}
f_{i g} & =-m_{i} \cdot g \\
f_{i b} & =V_{i}(t) \cdot \rho_{w} \cdot g \\
V_{i}(t) & =\frac{1}{2} \cdot\left[V_{0}+V_{C o m p r}(t)\right]+\Delta V_{u \_i}(t) \\
V_{C o m p r}(t) & =\gamma_{0} \cdot z(t)
\end{aligned}
$$

Donde $f_{g}=f_{1 g}+f_{2 g}$ es la fuerza de gravedad, $f_{b}=f_{1 b}$ $+f_{2 b}$ representa la fuerza de empuje, $g$ es la constante gravitatoria y $\mathrm{V}_{\mathrm{i}}(\mathrm{t})$ denotan volúmenes sumergidos para $\mathrm{i}=1,2$. Puesto que los accionamientos producirán variaciones de volumen, se consideran dependientes del tiempo. $\mathrm{V}_{0}$ representa el volumen nominal del cuerpo fuera del agua (sin compresión) y se asume constante. Finalmente $\gamma_{0}$ es el coeficiente de compresibilidad del cilindro, constante y positivo. Sólo se considera para valores negativos de la profundidad y nulo cuando el cuerpo no está sumergido. $\Delta \mathrm{V}_{\mathrm{u}_{\mathrm{i}}}(\mathrm{t})$ representa los volúmenes de control, mientras que $\mathrm{V}_{\text {Compr }}(\mathrm{t})$ expresa la pérdida de volumen por el propio efecto de la compresibilidad. Finalmente, $\rho_{\mathrm{w}}$ es la densidad del agua, considerada constante. Nótese que bajo flotabilidad neutra perfecta, $\mathrm{m}_{1}+\mathrm{m}_{2}=\rho_{\mathrm{w}} \mathrm{V}_{0}$ y que las fuerzas provocadas por los accionamientos son $\mathrm{f}_{\mathrm{i}}=\rho_{\mathrm{w}} \mathrm{g} \Delta \mathrm{V}_{\mathrm{u}_{-} \mathrm{i}}$ para $\mathrm{i}=$ 1,2 .

En cuanto a la dinámica de rotación, una vez obtenidas las condiciones de operación de equilibrio, bajo flotabilidad neutra, resulta:

$$
\left(I_{y y}+I_{y y \_} \text {Add }\right) \ddot{\theta}+\nu_{\theta} \cdot \operatorname{sign}(\dot{\theta}) \dot{\theta}^{2}=\left(f_{1}-f_{2}\right) \frac{L C_{\theta}}{2}+f_{2} \cdot \epsilon_{z} S_{\theta}
$$

Donde $S_{\theta}$ denota $\operatorname{sen}(\theta), C_{\theta}$ denota $\cos (\theta), I_{y y}$ es el momento de inercia con respecto al eje $\mathrm{y}, \mathrm{I}_{\mathrm{yy}_{-} \text {Add }}$ representa el efecto de la masa añadida en la inercia, $\mathrm{v}_{\theta}$ refleja el coeficiente de fricción, $\mathrm{L}$ es la longitud nominal del cilindro y $\varepsilon_{\mathrm{z}}$ indica el desplazamiento vertical según la Figura 3(b). La relación entre los desplazamientos de volumen provocados por los accionamientos y los pares generalizados proceden de la siguiente relación:

$$
\underbrace{\left[\begin{array}{c}
f_{z} \\
\Gamma_{y}
\end{array}\right]}_{\Xi}=\underbrace{\left[\begin{array}{c}
1 \\
\frac{L C_{\theta}}{2}-\frac{L C_{\theta}}{2}+\epsilon_{z} S_{\theta}
\end{array}\right]}_{\mathbf{M}} \cdot \underbrace{\rho_{W} \cdot g \cdot\left[\begin{array}{l}
\Delta V_{u_{-} 1} \\
\Delta V_{u_{2}}
\end{array}\right]}_{\mathbf{F}}
$$

Puede comprobarse que la matriz $\mathbf{M}$ es invertible para todo el rango $-\pi / 2 \leq \theta \leq \pi / 2$ debido al desplazamiento $\varepsilon_{\mathrm{z}}$ incluido en el modelo y mostrado en la Figura 3(b).

Por último, el diagrama de bloques del modelo dinámico presentado se ilustra en la Figura 4, y se puede expresar de forma matricial como sigue $\left\{\right.$ siendo $\left.\mathbf{q}(\mathrm{t})=[\mathrm{z}(\mathrm{t}) \theta(\mathrm{t})]^{\mathrm{T}}\right\}$.
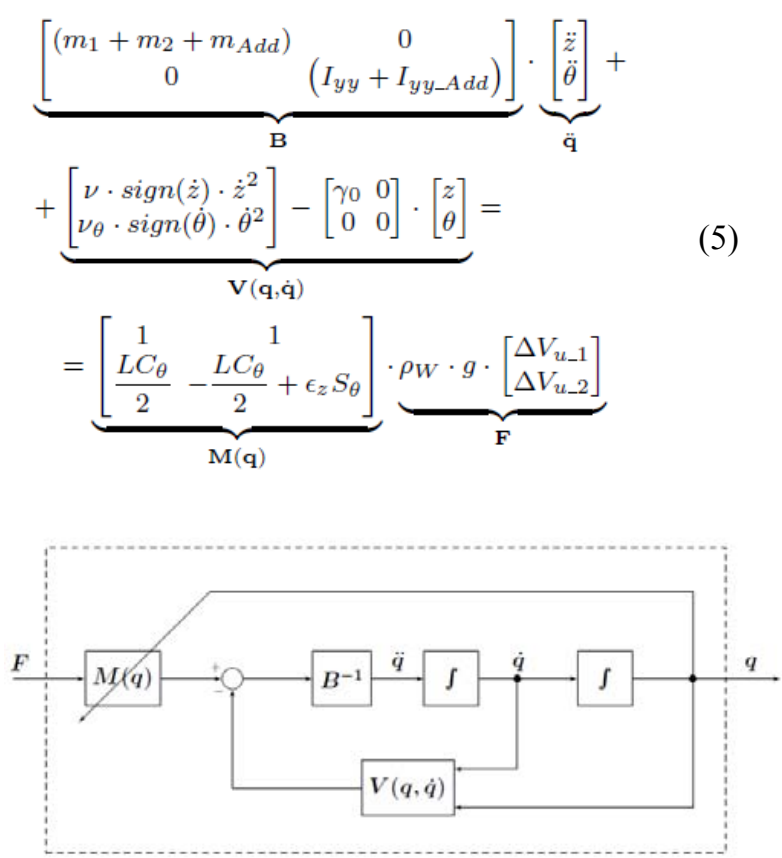

Figura 4. Modelo dinámico de dos grados de libertad.

\section{ALGORITMO DE CONTROL NO LINEAL}

Con el fin de seguir un desarrollo metodológico que resulte consistente con el diseño del algoritmo de control, se propone tratar el problema de control en el contexto de sistemas multivariables no lineales. Se utiliza el método directo de Lyapunov como base para diseñar un controlador basado en una transformación no lineal, una acción lineal tipo Proporcional-derivativa (PD) y un término no lineal de compensación diseñado para garantizar estabilidad global asintótica en la postura del TEC (véase, por ejemplo [9]). Si se define $\mathbf{q}_{\mathbf{d}}$ como el vector de las variables de salida deseadas y $\mathbf{e}_{\mathbf{q}}=\mathbf{q}_{\mathbf{d}}-\mathbf{q}$ denota el vector de error entre la postura deseada y la salida, la siguiente función definida positiva se selecciona como función candidata de Lyapunov:

$$
L\left(\dot{\mathbf{q}}, \mathbf{e}_{\mathbf{q}}\right)=\frac{1}{2} \dot{\mathbf{q}}^{T} \mathbf{B} \dot{\mathrm{q}}+\frac{1}{2} \mathbf{e}_{\mathbf{q}}^{T} \mathbf{K}_{\mathbf{p}} \mathbf{e}_{\mathbf{q}} \quad \forall \dot{\mathbf{q}}, \mathbf{e}_{\mathbf{q}} \neq \mathbf{0}
$$

Ecuación en la que $\mathbf{K}_{\mathbf{p}} \in \mathfrak{R}^{2 \times 2}$ es una matriz diagonal y definida positiva. Una política plausible con la que obtener la condición deseada $\mathbf{e}_{\mathbf{q}}=0$, es adoptar una acción de control $\mathrm{F}$, que dé como resultado un decrecimiento estricto de L. La acción de control ha de influir en el sistema realimentado de modo que resulte $\mathrm{dL} / \mathrm{dt}<0$. Diferenciando la ecuación (6) con respecto del tiempo y teniendo en cuenta que $\mathbf{q}_{\mathbf{d}}$ es constante, se obtiene: 


$$
\dot{L}\left(\dot{\mathbf{q}}, \mathbf{e}_{\mathbf{q}}\right)=\dot{\mathbf{q}}^{T} \mathbf{B} \ddot{\mathbf{q}}+\frac{1}{2} \dot{\mathbf{q}}^{T} \dot{\mathbf{B}} \dot{\mathrm{q}}-\dot{\mathbf{q}}^{T} \mathbf{K}_{\mathbf{p}} \mathbf{e}_{\mathbf{q}}
$$

Teniendo en cuenta que la matriz B también se mantiene constante, sustituyendo la expresión (5) en (7) y, reagrupando términos, es inmediato:

$$
\dot{L}\left(\dot{\mathrm{q}}, \mathbf{e}_{\mathbf{q}}\right)=\dot{\mathrm{q}}^{T}\left[-\mathbf{V}(\mathbf{q}, \dot{\mathrm{q}})-\mathbf{K}_{\mathbf{p}} \mathbf{e}_{\mathbf{q}}+\mathbf{M}(\mathbf{q}) \mathbf{F}\right]
$$

El término resultante es definido negativo. Asumiendo la perfecta cancelación de términos, a partir de (8) se elige la siguiente ley de control:

$$
\mathbf{F}=\mathrm{M}^{-1}(\mathbf{q})\left[\mathbf{V}(\mathbf{q}, \dot{\mathbf{q}})+\mathbf{K}_{\mathbf{p}} \mathrm{e}_{\mathbf{q}}-\mathbf{K}_{\mathrm{d}} \dot{\mathbf{q}}\right]
$$

Donde $\mathbf{K}_{\mathbf{d}} \in \mathfrak{R}^{2 \times 2}$ es una matriz diagonal definida positiva. La definición de la expresión (9) requiere la computación en línea del término no lineal $\mathbf{V}(\mathbf{q}, \dot{\mathbf{q}})$ así como la matriz $\mathrm{M}^{-1}(\mathbf{q})$, lo cual corresponde con una transformación no lineal de la entrada y la compensación de los términos de fricción y compresibilidad, junto con una realimentación lineal desacoplada de tipo PD. De hecho, sustituyendo la expresión (9) en (8), resulta:

$$
\dot{L}\left(\dot{\mathbf{q}}, \mathbf{e}_{\mathbf{q}}\right)=-\dot{\mathbf{q}}^{T} \mathbf{K}_{\mathbf{d}} \dot{\mathbf{q}}
$$

Ecuación que satisface $\dot{L}\left(\dot{\mathrm{q}}, \mathrm{e}_{\mathbf{q}}\right)<0$ y la función candidata $L\left(\dot{\mathbf{q}}, \mathbf{e}_{\mathbf{q}}\right)$ propuesta en (6) decrece mientras $\dot{\mathrm{q}} \neq 0$ para cualquier trayectoria. Puede verse que el sistema alcanza una postura de equilibrio. Para encontrar esta postura, nótese que $\dot{L}\left(\dot{\mathbf{q}}, \mathbf{e}_{\mathbf{q}}\right) \equiv 0$ si y solo si $\dot{\mathbf{q}} \neq 0$. La dinámica del sistema realimentado bajo el control nominal propuesto en (9) viene dada por:

$$
\mathrm{B} \ddot{\mathrm{q}}=\mathrm{K}_{\mathrm{p}} \mathrm{e}_{\mathrm{q}}-\mathrm{K}_{\mathrm{d}} \dot{\mathrm{q}}
$$

En condiciones de equilibrio, definidas por $\ddot{\mathrm{q}} \equiv 0 \mathrm{y}$ $\dot{\mathrm{q}} \equiv \mathbf{0}$, de (11) se obtiene que $\mathbf{K}_{\mathrm{p}} \mathbf{e}_{\mathbf{q}}=\mathbf{0}$, y por lo tanto, $\mathbf{e}_{\mathbf{q}}=\mathbf{q}_{\mathbf{d}}-\mathbf{q}=\mathbf{0}$ en la postura de equilibrio buscada. Resulta entonces global y asintóticamente estable el comportamiento del TEC con un control lineal de tipo PD y compensación de los términos no lineales. Puesto que las matrices de las ganancias $\mathbf{K}_{\mathbf{p}}$ y $\mathbf{K}_{\mathbf{d}}$ son definidas positivas, la estabilidad del sistema está garantizada para cualquier juego de valores de estas matrices. La Figura 5 muestra finalmente, el esquema de control resultante.

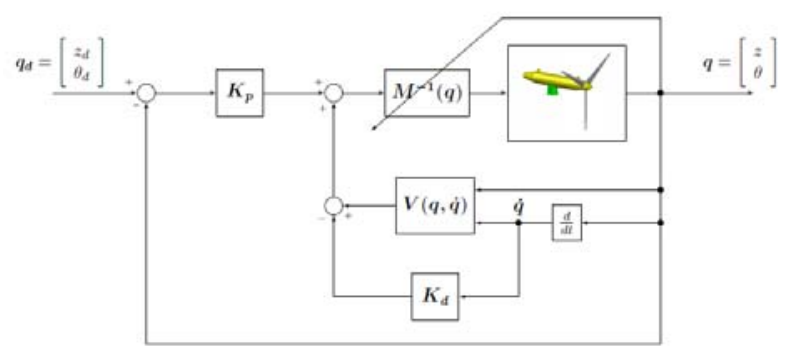

Figura 5. Sistema de Control.

\section{SIMULACIONES NUMÉRICAS}

El modelo dinámico propuesto y el sistema de control no lineal han sido implementados para llevar a cabo simulaciones numéricas con el fin de demostrar la bondad del desarrollo propuesto cuando el TEC lleva a cabo maniobras de emersión/inmersión. Los valores físicos del TEC de primera generación fueron: $\mathrm{m}_{1}=\mathrm{m}_{2}=38545[\mathrm{~kg}]$, $\mathrm{m}_{\text {Add }}=55119[\mathrm{~kg}], \mathrm{L}=16.8[\mathrm{~m}], \rho_{\mathrm{w}}=1025\left[\mathrm{~kg} / \mathrm{m}^{3}\right]$, $\mathrm{g}=9.81\left[\mathrm{~m} / \mathrm{s}^{2}\right], v=24830[\mathrm{~kg} / \mathrm{m}], v_{\theta}=18204[\mathrm{~kg} \mathrm{~m}]$ y $\varepsilon_{\mathrm{z}}=0.1[\mathrm{~m}]$. Se han utilizado señales de referencia lineales con tramos parabólicos [6],[14] que da lugar a perfiles trapezoidales de velocidad. Los parámetros de movimiento son los siguientes: $\theta_{\mathrm{d} 0}=0\left[^{\circ}\right], \theta_{\mathrm{df}}=90$ $\left[{ }^{\circ}\right], \mathrm{t}_{\theta 0}=5[\mathrm{~s}]$ y $\mathrm{t}_{\theta \mathrm{f}}=105[\mathrm{~s}] . \quad \mathrm{z}_{\mathrm{d} 0}=-20[\mathrm{~m}], \mathrm{z}_{\mathrm{df}}=-8$ $[\mathrm{m}], \mathrm{t}_{\mathrm{z} 0}=150[\mathrm{~s}] \mathrm{y} \mathrm{t}_{\mathrm{zf}}=225[\mathrm{~s}]$.

Eligiendo las ganancias de los controladores para que la respuesta transitoria del sistema realimentado ante entradas en escalón no ofrezca sobreoscilación alguna, siguiendo el método expuesto en la sección 3, los valores de los reguladores son: $\mathbf{K}_{\mathbf{p}}=\operatorname{diag}\{14$, $900\}$ y $\mathbf{K}_{\mathbf{d}}=\operatorname{diag}\{48.6,3142\}$. Los polos del sistema realimentado se sitúan en $-0.01 \mathrm{rad} / \mathrm{s}$ (polos dobles reales). El tiempo de muestro en la simulación ha sido de $10^{-1}$ [s]. Las respuestas de los estados de la dinámica del sistema no lineal obtenido para cada uno de los movimientos independientes del TEC se muestran en la Figura 6. Puede observarse que el sistema desarrolla un comportamiento deseado según las especificaciones de diseño en cuanto a su tiempo de establecimiento y su ausencia de sobreoscilaciones. Esta figura muestra también que el dispositivo se encuentra siempre completamente sumergido y no existe interacción con la superficie libre (nivel del mar). La Figura 7 muestra la evolución de las señales de control de volumen del sistema responsables de producir las fuerzas hidrostáticas requeridas para controlar la profundidad y orientación del dispositivo.

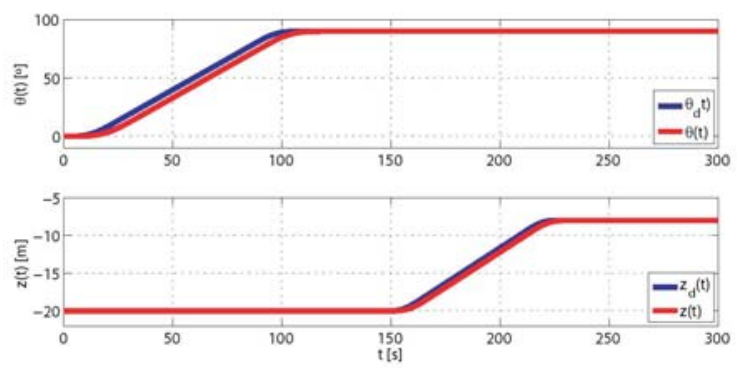

Figura 6. Maniobra de Emersión. Referencia y respuesta temporal.

Finalmente, para comparar las respuestas temporales del sistema controlado con el sistema de control propuesto, se ha simulado la respuesta de un sistema lineal desacoplado con polos en $-0.01[\mathrm{rad} / \mathrm{s}]$, la 
Figura 8 (izquierda) muestra ambas respuestas (la del sistema no lineal y el sistema ideal lineal de referencia) cuando una señal de escalón entre -40.0 y -27.5 $\mathrm{m}$ se utiliza como consigna para la profundidad. De la misma forma, la Figura 8 (derecha) muestra ambas respuestas (sistema no lineal realimentado y sistema ideal lineal) cuando una señal en escalón entre $0.0^{\circ}$ y $89.45^{\circ}$ se utiliza como consigna de orientación en escalón con instante de transición en $\mathrm{t}=400[\mathrm{~s}]$. En este conjunto de figuras, se suprimió la saturación de los accionamientos con el fin de mostrar el comportamiento del conjunto con señales del tipo escalón. Puede verse la gran concordancia en estos dos conjuntos de respuestas con las respuestas ideales esperadas.

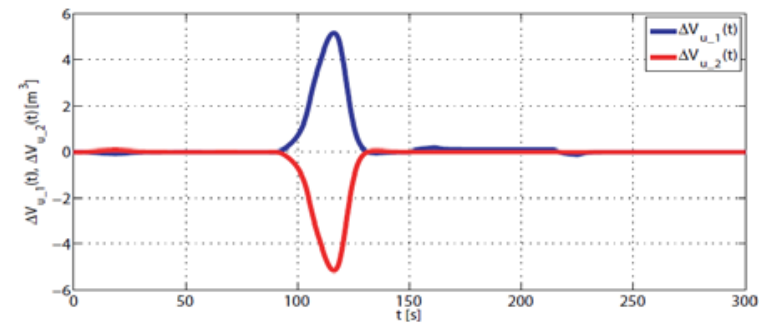

Figura 7. Maniobra de emersión. Evolución temporal de los volúmenes de control $\Delta \mathrm{V}_{\mathrm{u}_{-} 1} \mathrm{y} \Delta \mathrm{V}_{\mathrm{u}_{-} 2}$.

Por último, la Figura 9 ofrece la las respuestas ante las mismas referencias en escalón anteriores, si bien se ha utilizado el regulador lineal sin la compensación no lineal $\mathbf{V}(\mathbf{q}, \dot{\mathbf{q}})$ en lugar del regulador propuesto. El término no lineal $\mathrm{M}^{-1}(\mathrm{q})$ denominado matriz de desacoplamiento permanece conectado en ambos casos. Puede apreciarse claramente que la respuesta de la profundidad $\mathrm{z}(\mathrm{t})$ en el sistema con el controlador lineal exhibe un comportamiento más lento con mayor tiempo de establecimiento. La respuesta del ángulo picth $\theta(\mathrm{t})$ resulta muy similar a la obtenida anteriormente con el término de compensación no lineal y las diferencias entre ambas respuestas no resultan fácilmente apreciables.
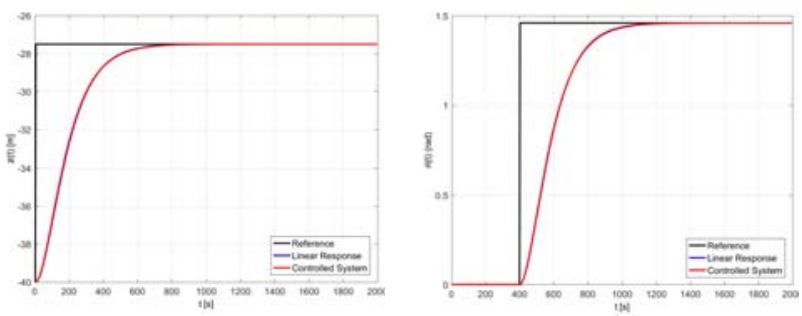

Figura 8. Comparación entre la respuesta obtenida y la esperada (lineal).
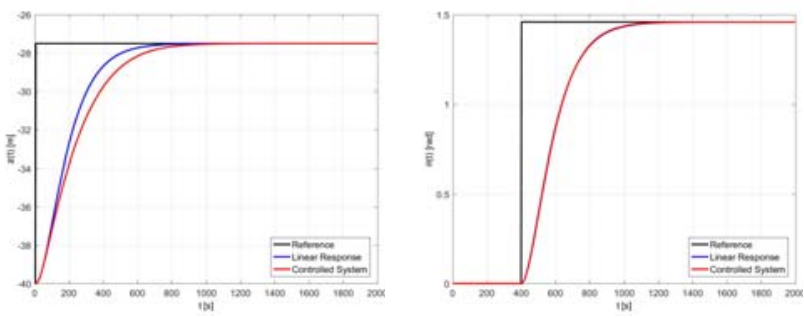

Figura 9. Comparación entre la respuesta obtenida con el controlador propuesto y con controlador lineal.

\section{CONCLUSIONES}

La explotación de energía de origen renovable de las mareas o de las corrientes oceánicas cuando la columna de agua es menor de 40 metros permite la instalación de los denominados dispositivos de primera generación. Se buscan nuevas soluciones a la automatización de maniobras de emersión/inmersión con el fin de mejorar su competitividad económica y de prestaciones. Las revisiones periódicas para mantenimiento son necesarias, durante las cuales el dispositivo tiene que ser extraído de la base en el lecho marino a la superficie libre (nivel del mar). Después de las intervenciones, la unidad de potencia debe ser devuelta a su base para retornar al modo de operación y extracción de energía. La automatización de estas maniobras debe ser considerada. La ejecución de estas operaciones se basa en el control en bucle cerrado del sistema mediante la gestión adecuada de agua de lastre contenida en su interior, por lo que la góndola habitual ha de ser modificada incluyendo estos tanques y sus adecuadas cámaras de máquinas para su gestión. Puesto que se requiere flotabilidad neutra del dispositivo con los tanques semi-llenos, las formas de la góndola han sido modificadas, manteniendo su diámetro y alargando su longitud. Además la distribución interior de los equipos de mayor peso ha de tenerse muy en cuenta para efectos de equilibrios hidrostáticos y estabilidad en flotación.

Este artículo propone un modelo dinámico muy simple que puede utilizarse para controlar un TEC de primera generación que ha sido concebido para extraer energía de las corrientes marinas. El modelo está basado en dos masas puntuales situadas en un plano. La ubicación de los accionamientos en los extremos del dispositivo permite dotar al mismo de dos grados de libertad. El control de estos dos grados de libertad se lleva a cabo mediante el manejo de agua de lastre contenida en tanques de la góndola, y que con su adecuada cámara de máquinas (bombas volumétricas y centrífugas, sistema de aire comprimido y valvulería diversa) permite producir fuerzas hidrostáticas de componente sólo vertical. Un algoritmo de control no lineal basado en la compensación de los términos de fricción y compresibilidad, junto con una matriz de 
desacoplamiento permite sintonizar reguladores por métodos habituales con los que ajustar los parámetros temporales como sobreoscilación y tiempo de establecimiento. Se obtienen respuestas adecuadas cuando se simulan maniobras de emersión e inmersión con sólo fuerzas hidrostáticas, pasivas. La estabilidad del sistema de control propuesto ha sido demostrada por el método directo de Lyapunov. Por último, se ha mostrado un leve análisis del comportamiento del dispositivo sin el término de compensación no-lineal obteniéndose también resultados satisfactorios. Se trabaja actualmente en el análisis del comportamiento de dispositivos sumergidos ante grandes incertidumbres paramétricas, sobre todo en lo que se refiere a la masa añadida, el efecto de señales de control fuertemente ruidosas $\mathrm{y}$ sistemas de filtrado $\mathrm{y}$ estimación paramétrica basado en técnicas algebraicas para la mejora de los sistemas de control para este tipo de dispositivos.

\section{Agradecimientos}

Este trabajo se ha realizado con financiación parcial del $\mathrm{M}^{\mathrm{o}}$ de Ciencia e Innovación, a través del Proyecto de Investigación Fundamental DPI2014-53499-R.

\section{English summary}

\section{DYNAMIC MODEL AND NONLINEAR CONTROL FOR A 2DOF FIRST GENERATION TEC}

\begin{abstract}
Several manufacturers have developed devices that exploit energy from marine currents in areas where the depth does not exceed 40 meters. These devices are called 1st Generation TEC. Maintenance tasks require extracting its gondola or nacelle from the base to the surface of the sea and returning to its base. It is possible to automate the emersion / immersion maneuvers and various works have been previously presented. This paper presents the simplest dynamic control model possible for a TEC handled by hydrostatic forces. A simple PD control system is proposed with the compensation of nonlinear terms that guarantee asymptotic and global stability, as well as a good behavior in movement.
\end{abstract}

Keywords: Tidal Energy Converters. Marine Renewable energy, Nonlinear Control.

\section{Referencias}

[1] Alstom Tidal Turbines Web Page. https://marineenergy.biz/tag/alstom/(Acc.2019)

[2] Andritz Hydro Hammerfest. https://www.andritz.com/hydroen/hydronews/hn-europe/unitedkingdom/video-hammerfest (Acc. 2019)

[3] Brito A. Villate J.L. Annual Report. Implementing agreement on Ocean Energy Systems. 2013. The Executive Committee of Ocean Energy Systems, 2014.

[4] Espín. M. Modelado dinámico y control de maniobras de dispositivos submarinos. Tesis Doctoral. Madrid. 2015.

[5] Hardisty J. The Analysis of Tidal Stream Power. Wiley. ISBN: 987-0-470-72451-4. 2009

[6] Hashimoto M., Oba F. and Nakahara H. Trajectory generation and tracking control methods for a multiple transfer robot system. Proc. IEEE/RSJ Intern. Workshop on Intelligent Robots and Systems (IROS 91), pp. 199-804.

[7] IEC-TC214. Technical specification: Marine energy. Wave, tidal and other water current converters. Part I: terminology, IEC/TS 626001 Ed. International Electrotechnic Commision, Ginebra, Switzerland, 2011.

[8] Portilla, M.P., López. A., Somolinos, J.A. y Morales R. Modelado Dinámico y Control de un Dispositivo Sumergido Provisto de Actuadores Hidrostáticos. RIAI. Vol. 15. No 1. Pg. 12-23. 2018.

[9] Sciavicco L. and Siciliano B. Modelling and Control of Robot Manipulators. Springer ISBN: 1-85233-221-2. 1999

[10] Siciliano B., Sciavicco L., Villani L. and Oriolo G. Robotics. Modelling, Planning and Control. Advanced Textbooks in Control and Signal Proccesing. Springer, ISBN 978-1-84996-6344. 2010

[11] SI Ocean. Ocean Energy: State of the Art. http://si-ocean.eu/en/upload/docs/WP3/ Technology Status Report FV.pdf 2012.

[12] SI Ocean. Ocean Energy: Cost of Energy and Cost Reduction Opportunities, Strategic Initiative for Ocean Energy. http://si-ocean.eu/en/upload/docs/WP3/CoE report 3 2 final.pdf, 2012.

[13] Verdant Power. Kinetic Hydropower System (KHPS). https://www.verdantpower.com/ (Acc. 2019).

[14] Saravana S. Jawahar N. Automated trajectory planner of industrial robot for pick-and-place task. International Journal of Advanced Robotic Systems, Vol. 10:100. 2013.

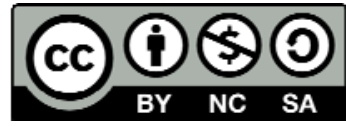
(C) 2019 by the authors. Submitted for possible open access publication under the terms and conditions of the Creative Commons Attribution CC BY-NC-SA 4.0 license (https://creativecommons.org/licenses/bync-sa/4.0/deed.es). 